\title{
Alterations in Grapevine Leaf Metabolism Occur Prior to Esca Apoplexy Appearance
}

\author{
Maryline Magnin-Robert, ${ }^{1}$ Marielle Adrian, ${ }^{2}$ Sophie Trouvelot, ${ }^{2}$ Alessandro Spagnolo, ${ }^{1}$ \\ Lucile Jacquens, ${ }^{2}$ Patricia Letousey, ${ }^{1}$ Fanja Rabenoelina, ${ }^{1}$ Mourad Harir, ${ }^{3}$ Chloé Roullier-Gall, ${ }^{4}$ \\ Christophe Clément, ${ }^{1}$ Philippe Schmitt-Kopplin, ${ }^{3,4}$ Armelle Vallat, ${ }^{5}$ Eliane Abou-Mansour, ${ }^{6}$ and \\ Florence Fontaine ${ }^{1, \dagger}$
}

${ }^{1}$ SFR Condorcet, Université de Reims Champagne-Ardenne, URVVC EA 4707, Laboratoire Stress, Défenses et Reproduction des Plantes, BP 1039, 51687 Reims Cedex 2, France; ${ }^{2}$ Agroécologie, AgroSup Dijon, CNRS, INRA, Univ. Bourgogne Franche-Comté, F-21000 Dijon, France; ${ }^{3}$ Analytical BioGeoChemistry, Helmholtz Zentrum München, German Research Center for Environmental Health, Neuherberg, Germany; ${ }^{4}$ Chair of Analytical Food Chemistry, Technische Universität München, Freising-Weihenstephan, Germany; ${ }^{5}$ Université de Neuchâtel, Institut de chimie, NPAC, Avenue de Bellevaux 51, 2000 Neuchâtel, Switzerland; and ${ }^{6}$ Plant Biology Department, University of Fribourg, Chemin du Musée 10, 1700 Fribourg, Switzerland

\section{Accepted 17 August 2017.}

Esca disease is one of the major grapevine trunk diseases in Europe and the etiology is complex, since several inhabiting fungi are identified to be associated with this disease. Among the foliar symptom expressions, the apoplectic form may be distinguished and characterized by sudden dieback of shoots, leaf drop, and shriveling of grape clusters in a few days that can ultimately induce the plant death. To further understand this drastic event, we conducted transcriptomic and metabolomic analyses to characterize responses of leaves during the period preceding symptom appearance ( 20 and 7 days before foliar symptom expression) and at the day of apoplexy expression. Transcriptomic and metabolomic analyses provide signatures for the apoplectic leaves and most changes concerning the metabolism of carbohydrates, amino acids, and phenylpropanoids. In deciphering glutathione-S-transferase (GST), its preferential location in phloem, correlated with the upregulation of GST genes and a decrease of the glutathione level, offers further support to the putative role of glutathione during apoplexy expression.

The 21 st century is experiencing a burst of re-emerging diseases, among them, the grapevine trunk diseases (GTDs), due to a complex of fungi. Over the past decade, their symptom incidence has drastically increased in Europe, i.e., the accumulation of symptomatic grapevines (foliar symptoms) in French vineyards is close to $13 \%$ (Bruez et al. 2013) and may reach up to $10 \%$ in Spain and 50\% in Italy (Romanazzi et al. 2009). A recent estimate of the economic cost of GTDs due to decreases in both quality and quantity of wine and the replacement of dead vines, evaluates the loss at more than 1.1 billion euros (Hofstetter et al. 2012). In Europe, GTDs are now considered the major obstacle for winegrowers, since no efficient control is available. Outside

\footnotetext{
${ }^{\dagger}$ Corresponding author: Florence Fontaine;
}

E-mail: florence.fontaine@univ-reims.fr

*The $\boldsymbol{e}$-Xtra logo stands for "electronic extra" and indicates that three supplementary tables are published online.

(c) 2017 The American Phytopathological Society
Europe, GTDs are also of great concern that induce, for example, economic losses of up to $\$ 260$ million per year in California and $\$ 8.3$ billion in Australia (De la Fuente et al. 2016).

Esca disease is one of the major GTDs in Europe and esca etiology is complex, since several fungi are known to be associated to this disease, especially Phaeomoniella chlamydospora, Phaeoacremonium minimum, and Fomitiporia mediterranea (Bertsch et al. 2013). They are xylem-inhabiting fungi and they induce various wood symptoms, such as brown necrosis and black streaking (Larignon et al. 2001). For foliar symptom expression, either a chronic or apoplectic form may be distinguished. The chronic form is typically characterized by spots appearing between the leaf veins or along the edges, which expand and become confluent, finally resulting in chlorotic and necrotic strips with only a narrow green stripe along the midrib (Bertsch et al. 2013; Mugnai et al. 1999). In most cases, the affected leaf finally assumes a "tiger stripe" appearance. The apoplexy form is characterized by the dieback of one or more shoots and is accompanied by leaf drop and the shriveling and drying of fruit clusters (Mugnai et al. 1999). Usually, this violent event occurs in midsummer, particularly when dry, hot weather follows rainfall (Mugnai et al. 1999; Surico et al. 2006). After such an event, the affected vines can resume growth in the following season or even in the current one but they can also ultimately die.

To further understand apoplexy expression, studies have been conducted on related grapevine responses in different organs (Fontaine et al. 2015). In the trunk, healthy wood was mainly characterized by underexpression of proteins involved in cell growth and defense response (Magnin-Robert et al. 2014). Conversely, in black-streaked wood, characterized by an extensive presence of GTD agents, the proteome analysis revealed overexpression of proteins involved in defense. For green shoots, various quantitative expressions are triggered upon the onset of apoplexy, although no pathogens associated with GTDs have been isolated from these organs (Spagnolo et al. 2012). In leaves, the period preceding symptom appearance was particularly investigated, by following physiological and targeted genes associated to photosynthesis and stress responses (Letousey et al. 2010). Within the week preceding symptoms, drastic alterations of photosynthesis were registered in preapoplectic vines, as 
revealed by a decrease in gas exchanges, changes in chlorophyll fluorescence, and repression of photosynthesis-related genes. In the meantime, expression of a set of defense-related genes was induced and amplified during symptom expression (Letousey et al. 2010). Such a characterization of the events preceding apoplexy needs new insights into gene regulation and metabolic changes associated with apoplexy expression.

In the present study, transcriptomic and metabolomic analyses were performed in grapevine 'Chardonnay' leaves, before and at the time of apoplexy appearance, in comparison with asymptomatic leaves as controls. This was completed by the quantitative reverse transcription-polymerase chain reaction (qRT-PCR) analysis of the expression of 18 targeted genes, selected from the previous transcriptomic study, on healthy and diseased samples collected 50,30, 20, and 7 days before apoplexy expression, focusing on glutathione metabolism and localization of the glutathione-S-transferase (GST), by in situ hybridization, to characterize its role in the detoxification process in response to the production of fungal toxins. The findings are discussed with respect to current knowledge about grapevine physiology and plant responses to fungal pathogen attacks.

\section{RESULTS}

Differentially expressed genes detected by Illumina mRNA before and at the time of the apoplexy event.

Analysis of gene expression was conducted on leaves from diseased plants at various times before apoplectic symptom appearance and on control plants at the same development stage. A total of $1,216,959$ raw reads were generated by 454 GS FLX sequencing of a cDNA mix of different leaf samples. After filtering, 1,210,565 clean reads were obtained, with an average length of $150 \mathrm{pb}$. The total length of clean reads was about 314 million bases $(314,141,226)$.

A selection of genes based on a differential expression between diseased and control leaves (ratio $\geq 2$ for upregulation and $\leq 0.5$ for downregulation) was done through all the timepoints. The total number of up- and downregulated genes was 2,122 . Overall, $51.8 \%$ of these genes showed a constant induction of their expression, $35.7 \%$ a constant repression, and $12.5 \%$ had an induction or a repression of their expression of at least two points of the kinetic. The evolution of the expression of the regulated genes (induced or repressed) depending on the kinetic was summarized in Figure 1. Results indicated that 262 genes exhibited a modified expression at 20 days before the apoplexy event (T0-20). The number of genes with regulated expression increased with the imminent onset of the symptoms, 629 genes for apparently visually healthy leaves 7 days before apoplexy (T0-7vh), 1,508 genes for turgorless leaves 7 days apoplexy (T0-7tg), and 1,690 genes at the onset of apoplexy (T0).
Interestingly, a majority of the genes with modified expression at T0-20 were down-regulated (60\%) (Fig. 1). This repartition was completely reversed on visually healthy leaves of diseased plants at T0-7, with 25\% downregulated genes. At the onset of symptoms, the number of down- or upregulated genes is equivalent. Genes regulated in diseased leaves were grouped into nine functional categories (Fig. 2), with about 69\% (181), $70 \%$ (440), 56\% (844), and 55\% (929) of the genes remaining, unfortunately, not yet known for categories T0-20, T0-7vh, T0$7 \mathrm{tg}$, and T0, respectively. Globally, from T0-20 to T0, there was an increasing number of regulated genes involved in both stress and defense reactions, lipid metabolism, and in protein metabolism and a decreasing number of genes involved in amino acid and secondary metabolism. Few modifications were observed for the other pathways. In regard to the nine functional categories reported-i) carbohydrate metabolism, ii) amino acid metabolism, iii) lipid metabolism, iv) growth, replication, and DNA damage repair, v) signalization, hormonal regulation, transduction, transcriptor factor, vi) protein metabolism, vii) cellular process, viii) secondary metabolism, and ix) stress and defense reaction (Supplementary Tables S2 and S3) - we focused on five representing key functions in grapevine physiology or already known to be affected by wood trunk diseases from previous studies, i.e., carbohydrate metabolism, amino acid metabolism, lipid metabolism, secondary metabolism, and stress and defense reactions (Koussa et al. 1998; Letousey et al. 2010; Lima et al. 2010; Magnin-Robert et al. 2011; Petit et al. 2006; Rifai et al. 2005). For carbohydrate metabolism, genes involved in energy synthesis, starch degradation, glucose metabolism, and photosynthesis system were globally up-regulated at the beginning of the kinetic (T0-20) in diseased leaves, before emergence of symptoms. Among them were the genes encoding succinate dehydrogenase (XM_002269371), lysosomalbeta-glucosidase (XM_002278327), zeta-carotene desaturase (XM_002277312), UTP-glucose 1-phosphate uridyltransferase (XM_002282240), alpha-glucan phosphorylase (XM_002280696), ribulose biphosphate carboxylase small chain (XM_002276931), and glucan endo-1,3-beta-glucosidase (XM_002279561). On the other hand, sucrose synthase 2 (XM_002271494), encoding a protein involved in sucrose synthesis was clearly repressed in the $\mathrm{T}-20, \mathrm{~T} 0-7 \mathrm{tg}$, and T0 categories, and two galactose oxidase genes (XM_01065799 and XM_002274727) encoding two proteins involved in galactose metabolism were clearly repressed in the T0-7tg and $\mathrm{T} 0$ categories. At T0, the upregulated genes were mainly involved in cell-wall formation and modification (beta-xylosidase/alpha-Larabinofuranosidase [XM_002264147], UDP-arabidopyranose mutase [XM_002263454]), whereas several genes involved in glycolysis, photosynthesis, and starch degradation were downregulated (violaxanthin de-epoxidase [XM_010650663]; alphaamylase [XM_002285177 and XM_0106655795]).

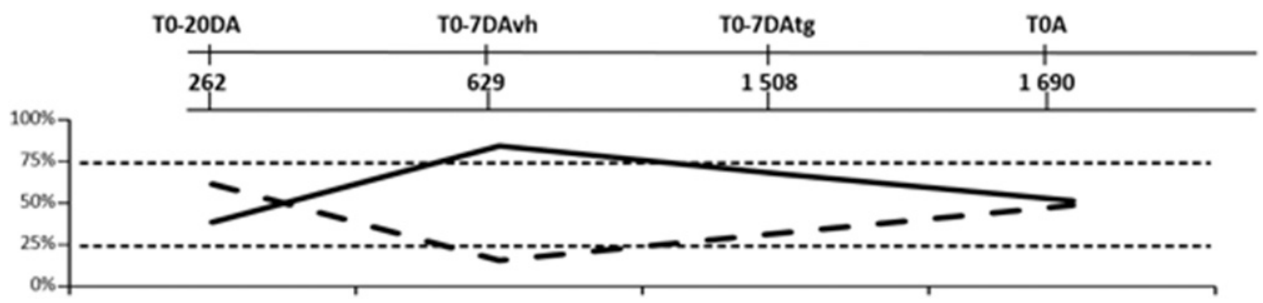

Fig. 1. Repartition of up- or down-regulated genes in grapevine leaf tissues ('Chardonnay') at 20 days and 7 days before appearance of symptoms and at the onset of apoplexy. The number of the genes differentially expressed in leaf tissues of future apoplectic plant in comparison with those of control (asymptomatic) plants for each timepoint is reported at the top. The expression of a same gene can be altered at different points of kinetics and, therefore, counted several times. The percentage indicates the repartition of genes characterized by an up- or a downregulation of their expression for each point of kinetic (solid or dotted line, respectively). T0-20 = visually healthy leaves sampled 20 days before the apoplexy event, T0-7vh = visually healthy leaves sampled 7 days before the apoplexy event, $\mathrm{T} 0-7 \mathrm{tg}=$ turgorless leaves sampled 7 days before the apoplexy event, and $\mathrm{T} 0=$ visually healthy leaves sampled at the onset of the apoplexy event. 
Genes involved in amino acid metabolism appeared to be also modulated during the kinetic, especially those involved in methionine and polyamine metabolism. For example, cysthathionine betalyase (XM_002281384), aspartate aminotransferase, mitochondrial (XM_002281728), thermospermine synthase (XM_002281075), tropinone reductase homolog (XM_002277799), S-adenosyl-Lmethionine decarboxylase (XM_002277280), and deoxyhypusine synthase (XM_002282885) were down-regulated, while S-adenosylL-methionine decarboxylase (XM_002278114) and asparte aminotransferase (XM_002284100) were up-regulated.

In lipid metabolism, changes were observed at both T0-7 and T0 timepoints, especially in category $\mathrm{T} 0-7 \mathrm{tg}$. There was an upregulation of genes associated with alkaloids and terpenoid synthesis, such as beta-amyrin synthase (XM_002270898), secologanin synthase (XM_003634726), VvCSbOciM (HM8807387), abietadienol/abietadienal oxidase (XM_010663730), hydroxymethylglutaryl-CoA synthase (XM_002282398), and 3epi-6-deoxocasthasterone 23 monooxygenase (XM_010650788). Conversely, the expression of 1-deoxy-D-xylulose-5-phosphate synthase (XM_002277883) and jmjC domain-containing protein 7 (XM_010646063) was down-regulated at the last three points of the kinetic, when the expression of alpha-terpineol synthase (XM_002266772) and alpha-terpineol synthase-like (XM_ 010660749) was down-regulated for both categories T0-7tg and T0. Some of the genes involved in the octadecanoid pathway were differently regulated, such as 12-oxophytodienoate reductase 2-like (XM_002281312) (upregulation at T0-7tg and T0) and allene oxide synthase (XM_002283744), lipoxygenase 1
(KF033130), and jasmonate-O-methyltransferase (XM 002281543) (downregulation, especially at $\mathrm{T} 0$ ).

Three genes related to phenylpropanoid metabolism were up-regulated at all timepoints, namely the salicylate-O-methyltransferase (XM_002262640), flavonoid-3'-monooxygenaselike (XM_0022842115), and bifunctional 3-dehydroquinate dehydratase/shikimate dehydrogenase (XM_002277359). Additionally, a downregulation of the expression of flavonoid-related R2R3MYB4 repressor transcription factor (XM_002278186) was observed at time T0-7. Several genes encoding proteins involved in anthocyanidin, flavonoid, and lignin biosynthesis were also up-regulated in categories T0-7tg and T0: malonate CoA (XM_002279103), anthocyanidin 3-O-glucosyl transferase (XM_002264962), UDP-glucoselflavonoid 3-O-glucosyltransferase (AB047093), chalcone synthase (XM_001280950), second bifunctional 3-dehydroquinate dehydratase/shikimate dehydrogenase (XM_010651497), cinnamyl alcohol dehydrogenase (XM_002285370), naringenin 2-oxoglutarate 3-dioxygenase (XM_002284856), and caffeoyl shikimate esterase (XM_003634810).

For the stress and defense reactions category, the epoxide hydrolase gene (XM_003632333), encoding a protein involved in the detoxification process, was up-regulated from T0-20 to T0. Except for the downregulation of the gene encoding a putative disease resistance RPP13, 12 genes encoding proteins involved in stress and defense reactions and in the detoxification process were up-regulated in leaves in categories T0-7vh, T0-7tg, and T0. Among them were genes encoding GST (XM_003634217 and XM_002262806) or haloacid dehalogenase (XM_002267523)
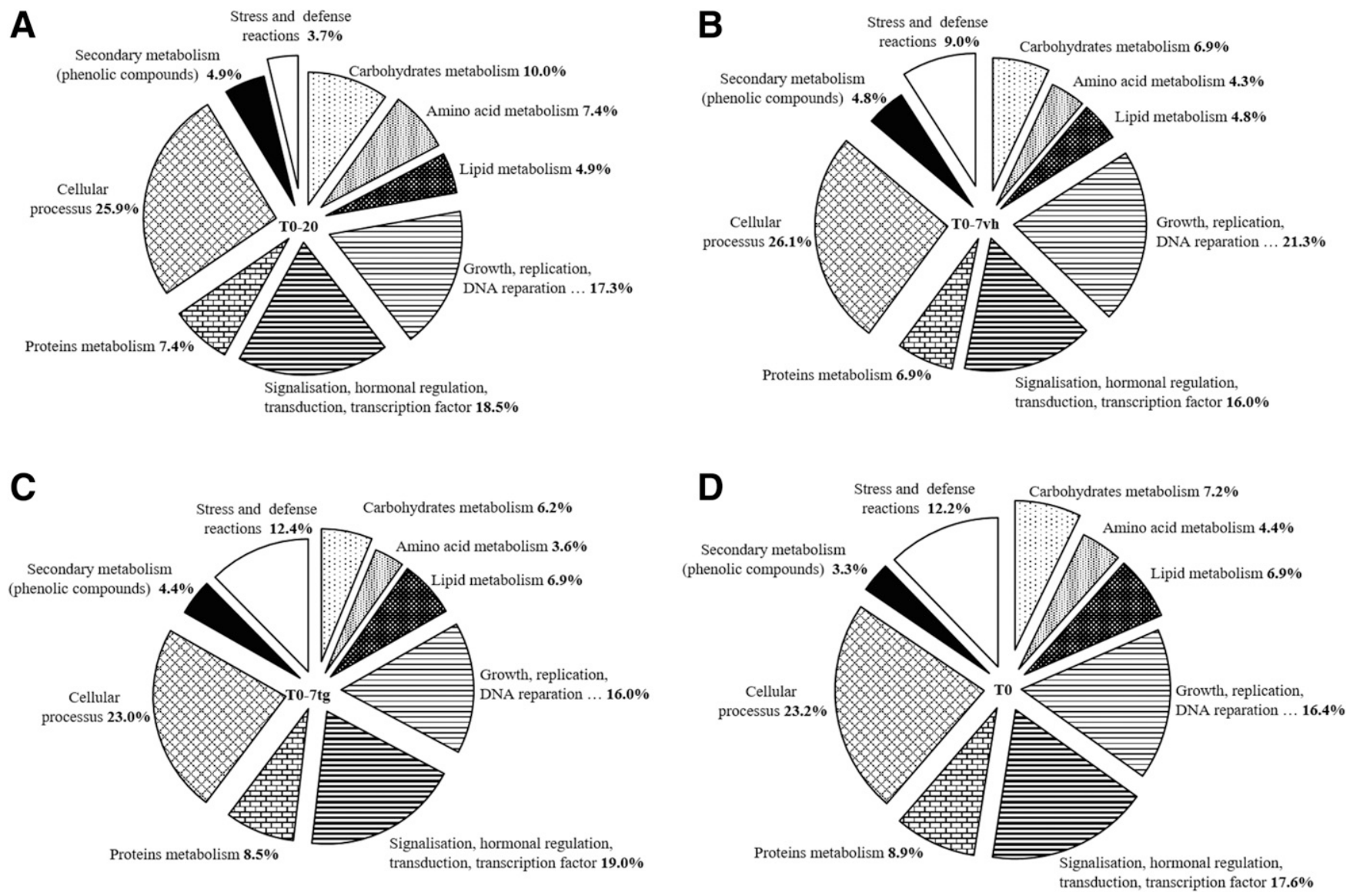

Fig. 2. Distribution into functional categories of genes differentially expressed in leaves of vines of 'Chardonnay' affected by the apoplexy event. The percentage of each category is reported in the figure. Only genes with a known function were integrated in this figure, which represents A, $31 \%$ of the total differentially expressed genes in visually healthy leaves sampled 20 days before the apoplexy event (T0-20), B, 30\% in visually healthy leaves sampled 7 days before the apoplexy event (T0-7vh), C, 44\% in turgorless leaves sampled 7 days before the apoplexy event (T0-7tg), and $\mathbf{D}$, 45\% in visually healthy leaves sampled at the onset of the apoplexy event (T0). 
and pathogenesis-related (PR) proteins such as thaumatin (XM_002274101), PR10 (XM_002273754), or class I $\beta$-1,3glucanase (XM_002277133). In categories T0-7tg and T0, the expression of genes related to multidrug transports, such as genes encoding pleiotropic drug resistance (XM_010655751, XM_010656419) and multidrug efflux transporter (XM_002274772, XM_002280176), was altered. At time T0-7, the expression of genes involved in the detoxification process was also regulated, as indicated by the induction of two epoxide hydrolase (GSVIVT00025835001, XM_010650793), the repression of two other GSTs (XM_002269082, XM_002275302), and one superoxide dismutase (XM_010661684). Moreover, a downregulation of chaperone protein dnaJ 20 (XM_002264118), hsp70-hsp90 protein 3 (XM_0036355638), chaperone protein ClpB4 (XM_002282844), and an upregulation for heat shock 70kDa (XM_0022834967) and 20-kDa chaperonin (XM_002277825) were reported. The expression of 24 genes encoding disease resistance proteins and 10 genes encoding PR proteins was also modulated for categories T0-7tg and T0.

\section{Validation of candidate genes by RT-PCR.}

Eighteen genes, selected on their expression pattern at three timepoints of the apoplexy event (Supplementary Table S1), were followed in leaves of diseased plants to validate these genes as precocious molecular markers of apoplexy expression. The samples used were the same ones as for the transcriptomic analyses, to which we added others obtained from diseased plants $(n=3)$ cultivated in the same vineyard during the same year. These genes were selected based on the regulation of their expression during the apoplectic kinetic (transcriptomic data at times T0-20 and T0-7) and their involvement in various pathways (carbohydrate metabolism, amino acid metabolism, lipid metabolism, hormonal regulation, cellular processes, phenylpropanoid pathway, and detoxification processes). Results correspond to means \pm standard deviation of three plants sampled. These genes code for UTP-glucose-1-phosphate uridyltransferase (gluPuriT-XM_002282240), sucrose synthase (SucS2-XM_002271494), succinate dehydrogenase flavoprotein (SucDHFla-XM_002269371), thermospermine synthase (SperS-XM_002281075), 1-deoxy-Dxylulose-5-phosphate (xylPS-XM_002277883), jmjC domaincontaining protein 7 (jmjc7-XM_010646063), auxin-induced protein 10A5 (Aux10A5-XM_002271727), myosin-H heavy chain (Myochain-XM_0022815579), F-box/WD40 repeatcontaining protein (FboxWD40-XM_002271652), casein kinase (CasK-XM_002284008), V-type proton ATPase subunit B (HatpZ-XM_002280255), flavonoid-related R2R3 MYB4a repressor transcription factor (MYB4A-XM_002278186), shikimate deshydrogenase (ShiDH- XM_002277359), epoxide hydrolase (epoxHF-XM_003632333), and haloacid dehalogenase hydrolase (Hahl-XM_002267523). Three of the four genes encoding a GST identified by the transcriptomic study were also selected: GSTA (XM_003634217), GSTB (XM8002262806), and GSTC (XM8002269082). No significant regulation of the selected genes involved in protein phosphorylation (cellular processes), amino acid metabolism, lipid metabolism, and transports was observed in our samples, suggesting they might be not retained as markers of the preapoplectic event. Among the three selected genes involved in carbohydrate metabolism, only $\mathrm{SucS} 2$ was down-regulated on leaves at time T0-20. Similar to the results of the transcriptomic analysis, Aux10A5 was down-regulated at two timepoints, T0-7 and T0 (Table 1). Moreover, the repression of MYB4A expression at T0-7 and T0 was confirmed, whereas no modifications were monitored at T0-20, T0-30, and T0-50. For the detoxification process, EpoxHF was up-regulated at T0 and Hahl was downregulated at timepoint T0-30 and then up-regulated at timepoints T0-7 and T0. Concerning the GST-encoding genes, GSTC expression was not modified during the kinetic, whereas GSTA and GSTB were down-regulated at T0 for GSTA and at T0 and T0-7 for
GSTB (Table 1). Moreover, these two genes appeared to be upregulated, in our conditions, 50 days before the apoplexy event (Table 1).

\section{Changes in the metabolome during the apoplexy event expression.}

Fourier-transform ion cyclotron resonance mass spectrometry (FT-ICR-MS) was used in the negative-ion electrospray ionization (ESI) mode for the nontargeted analysis of the lowmolecular weight metabolites present in the sample sets. A total of 40,149 raw mass values was obtained and reduced for masses occurring in a minimum of $10 \%$ of all samples and by the calculation of elemental formulas with NetCalc software (Tziotis et al. 2011). The dataset containing 7,309 masses assigned to an elementary composition was thus obtained, among which, only those with $1,255 \mathrm{~m} / z$ could be annotated to putative metabolites after querying Kyoto Encyclopedia of Genes and Genomes (KEGG), HMDB, and LipidMaps databases. Orthogonal projections to latent structures-discriminant analysis (OPLS-DA) allowed the discrimination of three sample groups with a good statistical significance, i.e., control, T0-20/T0-7, and T0 diseased samples (Fig. 3A). Among the 7,309 elementary compositions obtained, 4,220 were common to the three groups whereas 1,206 were specific to control, 87 to diseased T0-20/T0-7 samples, and 32 to diseased T0 samples (Fig. 3B). The chemical composition and van Krevelen (VK) diagrams obtained from all $\mathrm{m} / \mathrm{z}$, highlighted a wide chemical diversity, with a significant contribution of $\mathrm{CHO}$ and, to a lesser extent, of CHONS elemental formulas (Fig. 3C). Masses with the highest regression coefficient values in each sample group (specific and common masses with a variable importance in projection [VIP] value $>1$, thereafter called 'top $\mathrm{m} / \mathrm{z}$ ') were next used for comparison, allowing the visualization of clear differences between the three sample groups (Fig. 3D, E, and F). CHO elemental formulas remained the most abundant in all groups, followed by CHONS and $\mathrm{CHON}$ formulas (Fig. 3D, E, and F, chemical composition diagrams on the right). $\mathrm{CHO}$ and $\mathrm{CHON}$ formulas were more abundant in diseased $\mathrm{T} 0$ samples, whereas $\mathrm{CHO}$ and CHONS were more abundant in control formulas. VK diagrams highlighted noteworthy differences in the nature and the number of formulas assigned to masses between the three sample groups, leading to specific fingerprints (Fig. 3D, E, and F).

The KEGG query (Vitis vinifera organism) with the MassTRIX interface allowed the assignment of top $\mathrm{m} / \mathrm{z}$, values to metabolic pathways. A rather limited number of pathways were associated to diseased samples, especially for T0-20/T0-7 (Fig. 4, six pathways). For this sample group, pathways were related to amino acid metabolism (tyrosine, phenylalanine, cysteine, and methionine), biosynthesis of secondary metabolites, flavone and flavonol biosynthesis, ubiquinone, and other terpenoid-quinone biosynthesis. In diseased T0 samples, more pathways were identified $(>10)$. The main ones were biosynthesis of secondary metabolites, starch and sucrose metabolism, galactose metabolism, phenylpropanoid biosynthesis, flavone and flavonol biosynthesis, flavonoid biosynthesis, monoterpenoid biosynthesis, phenylalanine metabolism, and oxocarboxylic acid metabolism (Fig. 4). The number of pathways associated to the control samples was even greater $(>18)$, and the main ones were biosynthesis of secondary metabolites, starch and sucrose metabolism, and flavonoid biosynthesis, galactose metabolism, and amino sugar and nucleotide sugar metabolism (Fig. 4).

A mass difference network analysis based on series of biochemical transformations (Adrian et al. 2017; Moritz et al. 2017) was performed to obtain an additional overview of the global metabolism in the different grapevine leaf groups (data not shown). A total of 469 transformations were found to connect masses. Those having the highest z-score were represented in the histogram in Figure 5. Four types of differences allowed discrimination 
between samples: opposite z-scores for diseased samples at T0, opposite $\mathrm{Z}$-scores for diseased samples at T0-7 and T0-20, higher z-scores of control samples, higher z-scores of diseased samples either at T0-7 and T0-20 or T0 times. Transformations in T0 samples were highly different from those of the two other sample groups, with the absence of biochemical reactions involving the conjugation of pteridine, phenylpyruvic, and coumaric acids, and an increased number of reactions involving phosphorylation, phosphoric acid addition and ring opening, decarboxylation, triprenylation/farnesylation, and also, linoleic acid metabolism and condensation (Fig. 5).

\section{Dynamics of glutathione during the apoplexy event expression.}

Attention was paid to the dynamics of glutathione in the samples. The query in the KEGG database (Vitis vinifera organism) with the MassTRIX interface first confirmed the importance of the glutathione pathway in diseased leaves (Fig. 6A); the $m / z=306.07655$ correspond to the formula $\left[\mathrm{C}_{10} \mathrm{H}_{17} \mathrm{~N}_{3} \mathrm{O}_{6} \mathrm{~S}\right]^{-}$, confirmed with the isotopologues. The signal intensity of putative glutathione in the different sample series was next assessed using the peak intensities of the spectra. Independently of the kinetic, the amount of this metabolite was about three times lower in apoplectic than in control samples (Fig. 6B). No mass corresponding to the reduced form of glutathione could be found. A metabolic network was built from S-containing compounds (41.07\% of all masses) and allowed the visualization of the central role of $\left[\mathrm{C}_{10} \mathrm{H}_{17} \mathrm{~N}_{3} \mathrm{O}_{6} \mathrm{~S}\right]^{-}$and metabolic connectivity (Fig. 6C). Masses containing 1, 2, 3, or $4 \mathrm{~S}$ represented 20.32, $16.74,3.60$, and $0.41 \%$ of the total masses, respectively. Masses associated to glutathione could be extracted from this network (Fig. 6D). Most of them were common to the three sample groups and were mainly condensations and additions (Fig. 6E).

\section{In situ hybridization visualization of GST1 expression in leaves.}

Since apparent modification of glutathione metabolism was observed with transcriptomic and metabolomic analysis, GST1 was further analyzed and located in plant tissues. GST1 has been reported to be induced in presymptomatic and symptomatic leaves, green shoots, cordon, and trunk of diseased grapevine affected by grapevine leaf stripe diseases or apoplexy external symptoms appearance (Letousey et al. 2010; Magnin-Robert et al. 2011, 2016). Moreover, the expression pattern of GST1 was monitored by RTPCR at T0 (36), T0-7 (2.99), T0-20 (3.8), T0-30 (1.91), and T0-50 (2.9), revealing that $G S T 1$ was up-regulated during this apoplexy kinetic in grapevine leaves, except at T0-30. To analyze in depth the cell type-specific expression of GST1, in situ hybridization experiments were performed in leaves of control or preapoplectic grapevines (Figs. 7 and 8). The results revealed a signal

Table 1. Relative expression of 18 selected genes in the visually healthy leaves of preapoplectic vines of 'Chardonnay'a

\begin{tabular}{|c|c|c|c|c|c|c|c|c|}
\hline \multirow[b]{2}{*}{ Functional classification } & \multirow[b]{2}{*}{ Selection as marker } & \multirow[b]{2}{*}{ Gene } & \multicolumn{5}{|c|}{ Relative expression of targeted genes ${ }^{b}$} & \multirow[b]{2}{*}{ Methods } \\
\hline & & & T0-50 & T0-30 & T0-20 & T0-7vh & T0 & \\
\hline \multirow[t]{6}{*}{ Carbohydrate metabolism } & No & gluPuriT & 1.07 & 1.01 & 1.23 & 0.72 & 1.26 & RT-PCR \\
\hline & & & & & 0.50 & 12.50 & 87.60 & Illumina \\
\hline & No & SucS2 & 0.92 & 0.88 & 0.36 & 0.56 & 0.72 & RT-PCR \\
\hline & & & & & 0.20 & 1.50 & 0.00 & Illumina \\
\hline & No & SucDHFla & 1.11 & 1.09 & 1.00 & 0.80 & 0.88 & RT-PCR \\
\hline & & & & & 5.10 & 13.50 & 4.70 & Illumina \\
\hline \multirow[t]{2}{*}{ Amino acid metabolism } & No & SperS & 1.81 & 1.29 & 1.29 & 0.97 & 0.57 & RT-PCR \\
\hline & & & & & 0.00 & 0.90 & 1.20 & Illumina \\
\hline \multirow[t]{4}{*}{ Lipid metabolism } & No & xylPS & 1.16 & 0.79 & 1.12 & 0.78 & 1.20 & RT-PCR \\
\hline & & & & & 1.30 & 0.50 & 0.20 & Illumina \\
\hline & No & jmjc7 & 1.00 & 1.07 & 1.27 & 0.70 & 0.71 & RT-PCR \\
\hline & & & & & 0.80 & 0.30 & 0.00 & Illumina \\
\hline \multirow[t]{6}{*}{ Hormonal regulation, signalization } & Ok & Aux10A5 & 1.25 & 0.48 & 1.60 & 0.29 & 0.22 & RT-PCR \\
\hline & & & & & 1.10 & 0.40 & 0.20 & Illumina \\
\hline & No & Myochain & 1.59 & 1.26 & 1.14 & 0.88 & 0.80 & RT-PCR \\
\hline & & & & & 9.20 & 9.30 & 2.10 & Illumina \\
\hline & No & FboxWD40 & 0.69 & 0.76 & 0.88 & 0.80 & 1.02 & RT-PCR \\
\hline & & & & & 4.20 & 1.20 & 51.20 & Illumina \\
\hline \multirow[t]{4}{*}{ Cellular processes } & No & CasK & 1.08 & 0.93 & 1.10 & 0.96 & 1.05 & RT-PCR \\
\hline & & & & & 3.90 & 0.90 & 0.10 & Illumina \\
\hline & No & HatpZ & 1.27 & 0.93 & 0.91 & 1.25 & 0.97 & RT-PCR \\
\hline & & & & & 3.20 & 11.40 & 61.30 & Illumina \\
\hline \multirow[t]{4}{*}{ Phenylpropanoid metabolism } & No & ShikDH & 0.96 & 0.85 & 0.83 & 1.23 & 1.11 & RT-PCR \\
\hline & & & & & 2.30 & 8.70 & 8.50 & Illumina \\
\hline & Ok & MYB4A & 0.64 & 1.25 & 0.81 & 0.41 & 0.27 & RT-PCR \\
\hline & & & & & 1.70 & 0.20 & 0.10 & Illumina \\
\hline \multirow{10}{*}{ Detoxification } & No & ерохHF & 0.98 & 1.06 & 1.71 & 1.42 & 2.29 & RT-PCR \\
\hline & & & & & 2.30 & 8.60 & 46.70 & Illumina \\
\hline & Ok & $H A L H$ & 1.40 & 0.46 & 1.73 & 2.49 & 3.23 & RT-PCR \\
\hline & & & & & 0.70 & 19.90 & 49.10 & Illumina \\
\hline & No & GSTA & 2.91 & 1.66 & 1.63 & 0.62 & 0.26 & RT-PCR \\
\hline & & & & & 1.00 & 14.70 & 56.50 & Illumina \\
\hline & No & GSTB & 3.94 & 1.90 & 1.11 & 0.26 & 0.21 & RT-PCR \\
\hline & & & & & 1.10 & 10.10 & 42.90 & Illumina \\
\hline & No & GSTC & 1.09 & 1.31 & 1.01 & 1.37 & 1.35 & RT-PCR \\
\hline & & & & & 1.10 & 0.60 & 0.30 & Illumina \\
\hline
\end{tabular}


preferentially located in veins (Fig. 7G), especially in preapoplectic vines. None or only a slight signal was detected in leaf tissues of control vines (Fig. 7E). Visualization of the GSTI transcripts matched with the cells of phloem complexes in the secondary bundles (Fig. 7G, red arrow heads), and was exclusively revealed with the antisense probe (Fig. $7 F$ and G). Magnification of the principal vein (primary bundles) hybridization allowed the localization of GST1 expression in the phloem complexes of preapoplectic leaves (Fig. 8G). Again, no (or only a slight) signal was detected in primary veins of asymptomatic leaves (Fig. 8E). In comparison with the Rib antisense probe
(Figs. 7C and 8C), no signal was detected in the epidermis, parenchyma, and xylem cells for the GST1 gene, thus confirming the preferential phloem-specific expression of the GST1 gene.

\section{DISCUSSION}

Esca disease is complex, not only because several fungi are causal agents but also because pathogens are localized in xylem vessels and fibers of mature wood, whereas visible symptoms appear at the foliar level in July and August (Bertsch et al. 2013; Larignon et al. 2001). Apoplectic form has a serious
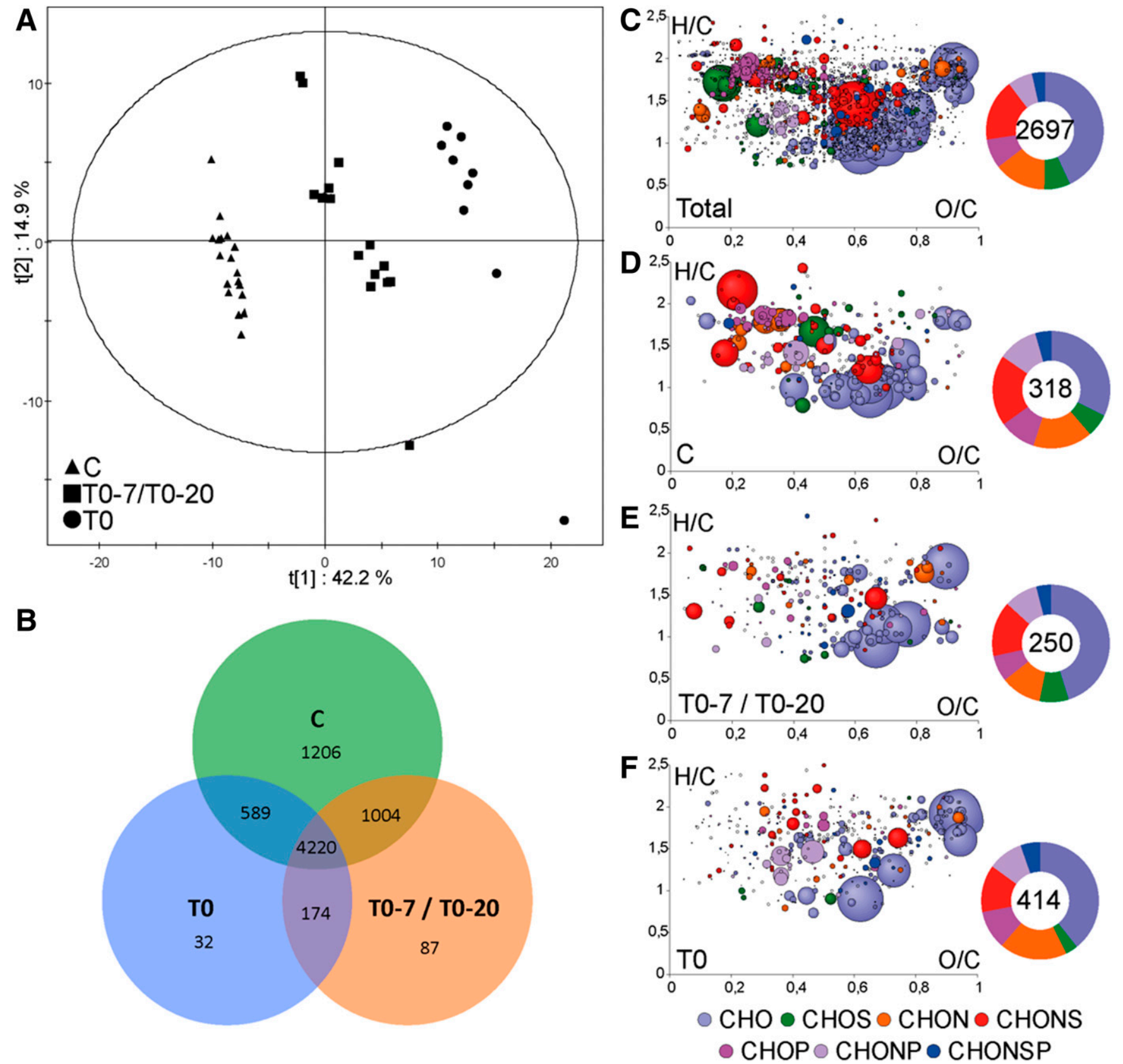

Fig. 3. Discrimination of Fourier-transform ion cyclotron resonance mass spectrometry (FT-ICR-MS) data samples and detailed visualization of the samples. A, Orthogonal projections to latent structures-discriminant analysis showing the discrimination of control, T0-20/T0-7 (leaves sampled 20 and 7 days before apoplexy), and T0 (time of the apoplexy event) diseased samples. B, Venn diagram showing the distribution of the $m / z$ obtained by (-) FT-ICR-MS analysis of the samples. C, Van Krevelen diagrams representing the elemental formulas attributed to the top $m / z$ of total samples, D, control, E, T0-20/T0-7 (leaves sampled 20 and 7 days before apoplexy), and F, T0 (time of the apoplexy event) samples onto two axes according to H/C and O/C atomic ratios. Dots are shaded according to their elemental composition (CHO, CHOS, CHON, CHONS, CHOP, CHONP, CHONSP) and are sized according to their relative intensity in mass spectra. Diagrams (on the right) indicate the numbers of these formulas and show their distribution according to their elemental composition (CHO, $\mathrm{CHOS}$, CHON, CHONS, CHOP, CHONP or CHONSP). Top $m / z$ values correspond to $m / z$ with the highest regression coefficient value (VIP $\geq 1$ ). 
impact on the vineyard safeguard, since it leads quickly to the death of the plant, and its incidence is increasing, partly due to climate fluctuation. To further understand the drivers leading to this status, the leaf responses before symptom emergence and at the onset of symptoms were particularly followed. More precisely, to follow the chronology of the onset of apoplexy appearance, leaves were sampled at 50,30, 20, and 7 days preceding the apoplexy event, with a focus from T0-20 to the day of expression (T0).

Our study clearly demonstrates changes in plant metabolism from T0-20. Transcriptomic analyses revealed an increase in the number of genes whose expression was altered as the apoplexy event was close: less than 300 genes at T0-20 to about 1,700 at T0. Modifications were mainly reported in the metabolisms of carbohydrates, amino acids, lipids, phenylpropanoids, and stress and defense responses. The expression of carbohydrate genes involved in energy synthesis, starch degradation, and photosynthesis was induced at T0-20 and was then repressed at T0, while those involved in plant defense response were up-regulated from
T0-7 to T0. This is in agreement with previous results obtained on leaves developing various GTDs (Camps et al. 2010; Letousey et al. 2010; Lima et al. 2010). Regarding amino acid metabolism, especially methionine and polyamine, an alteration was observed during the kinetic, as described in leaves affected by Eutypa dieback (Rifai et al. 2005). This metabolic pathway could be crucial for grapevine immune responses toward the pathogens, as observed for grapevine response to Botrytis cinerea (Hatmi et al. 2013). In addition, the grapevine response is strengthened by an induction of defense genes, especially related to the phenylpropanoid pathway during the apoplexy emergence, which are generally up-regulated following a pathogen attack, including those involved in trunk diseases (Calzarano et al. 2014; Lambert et al. 2013; Lima et al. 2010; Magnin-Robert et al. 2011; Valtaud et al. 2009) and other diseases (Gruau et al. 2015; RomeroPérez et al. 2001).

In the meantime, a metabolic signature of apoplectic leaves was observed. FT-ICR-MS was used to analyze and compare the metabolome of the different leaf samples (the same as those
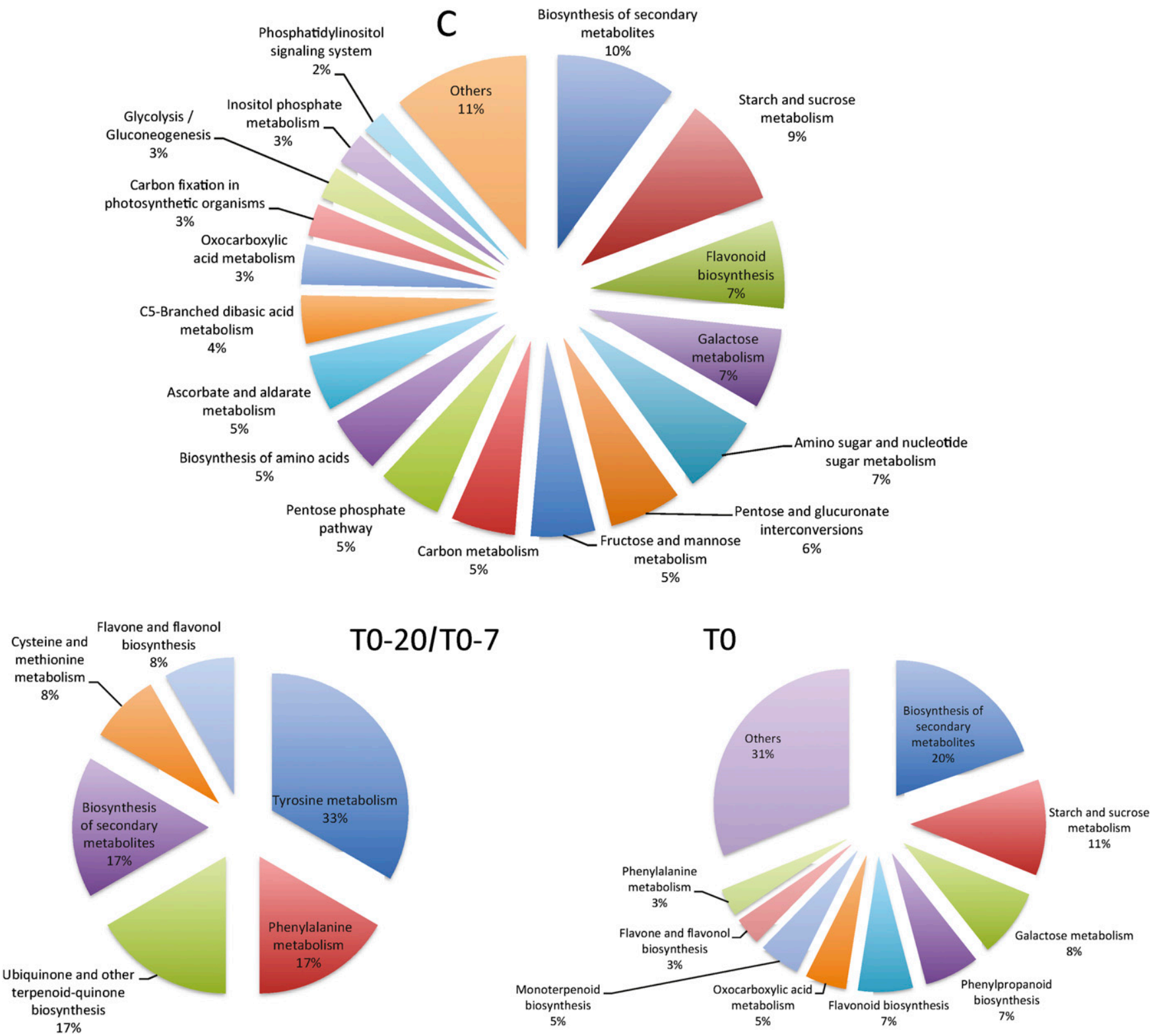

Fig. 4. Metabolic pathways associated to the top $\mathrm{m} / \mathrm{z}$ identified in the different sample groups. The metabolic pathways corresponding to the top $\mathrm{m} / \mathrm{z}$ of control (C), T0-20/T0-7 (leaves sampled 20 and 7 days before apoplexy), and T0 (time of the apoplexy event) samples were obtained after a Kyoto Encyclopedia of Genes and Genomes query with the MassTRIX interface, using Vitis vinifera organism. 
used for transcriptomic analysis). OPLS-DA allowed the distinction of three sample groups: samples from asymptomatic leaves (control), samples of preapoplectic diseased leaves (T020/T0-7), and samples of leaves at the time of apoplexy expression (T0). The leaf metabolome was therefore affected by esca disease and in a different manner before and at the time of apoplexy expression. A Venn diagram revealed the occurrence of specific $m / z$ in diseased leaves ( 87 for T0-20/T0-7 and 32 for T0 samples), suggesting that either new compounds accumulated before and at the time of apoplexy expression in diseased

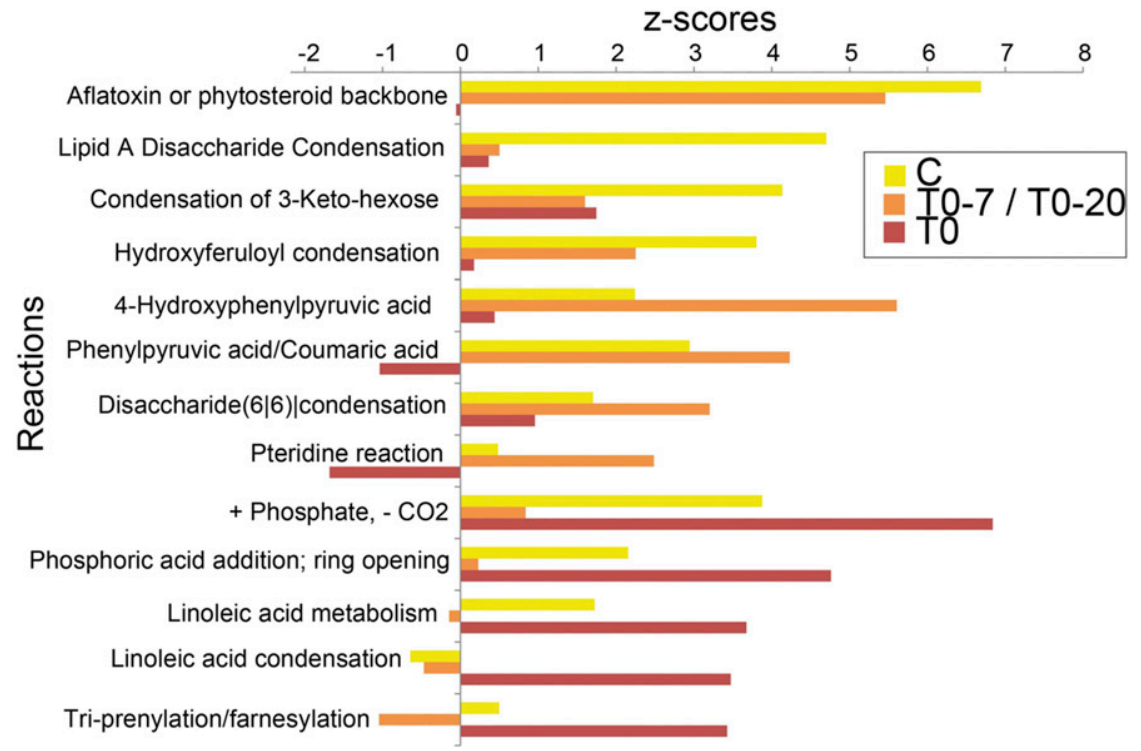

Fig. 5. Possible transformations of the complete $\mathrm{m} / \mathrm{z}$ data set.

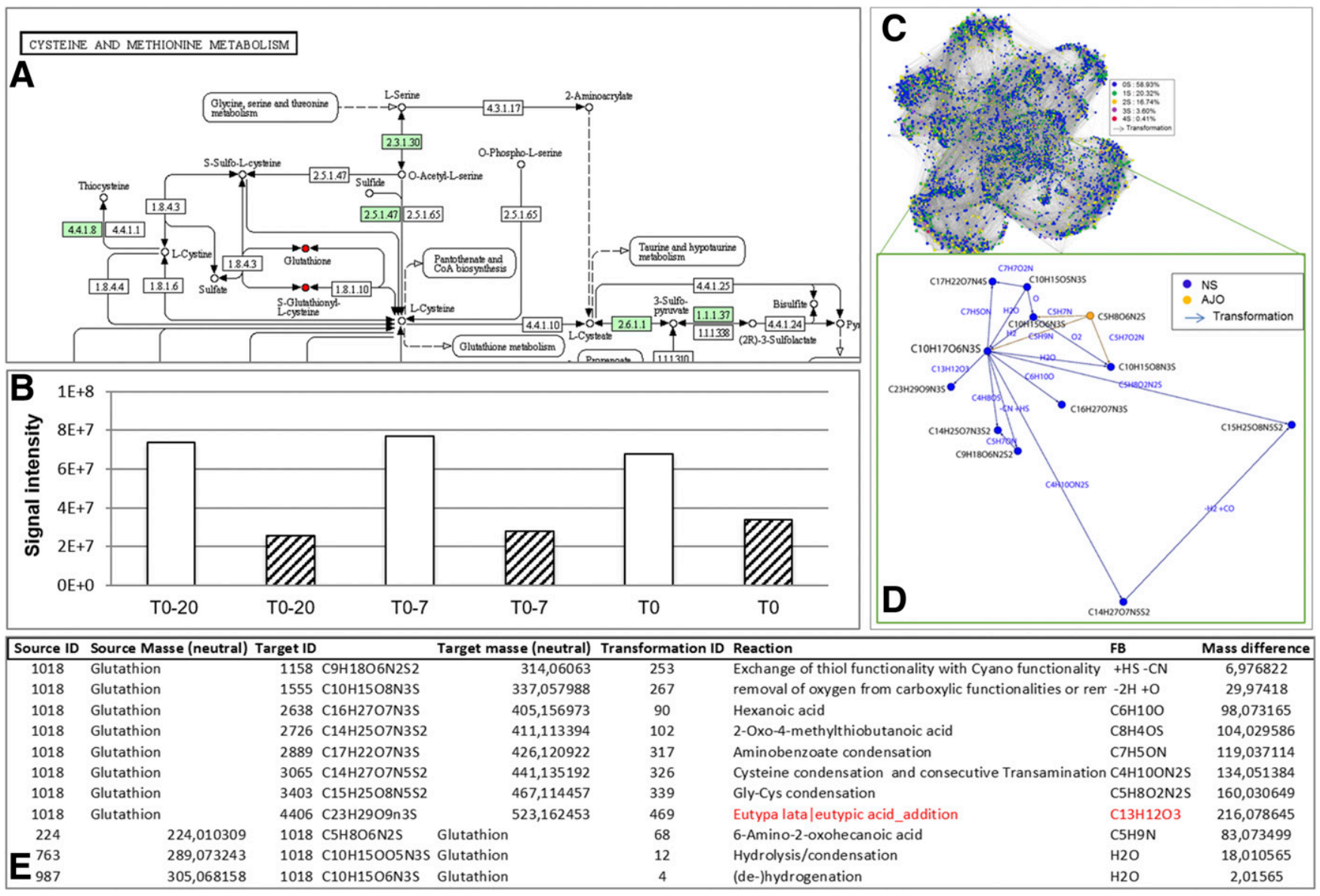

Fig. 6. Focus on glutathione metabolism. A, Partial metabolic pathway obtained after a Kyoto Encyclopedia of Genes and Genomes query with the MassTRIX interface, using the Vitis vinifera organism, focused on glutathione metabolism, B, signal intensity of putative glutathione in control (white bars) and diseased (striped bars) samples, $\mathbf{C}$, network analysis of $\mathbf{S}$ containing compounds with a focus on $\mathbf{D}$, glutathione and $\mathbf{E}$, an example of transformations involving glutathione. 
samples or that compounds initially present were metabolized during symptom appearance, or both. The lower number of these specific $\mathrm{m} / \mathrm{z}$ at T0 than at T0-20 and T0-7 may be due to apoplexy expression (stop of the metabolism and sap movement). Moreover, both apoplectic sample groups have a lower number of specific $\mathrm{m} / \mathrm{z}$ than the control, indicating a reduced diversity of the metabolic composition.

VK diagrams obtained from discriminant masses provided a metabolic fingerprint for each of the three distinct groups. KEGG query (Vitis vinifera organism) with the MassTRIX interface allowed the association of amino acid metabolism (tyrosine and phenylalanine), biosynthesis of secondary metabolites, ubiquinone and other terpenoid-quinone biosynthesis pathways to the T0-20/T0-7 sample group. The amino acid phenylalanine plays a crucial role as the precursor of the phenylpropanoid pathway leading to the biosynthesis of lignin, stilbenes, and flavonoids. All these compounds are known to play a role against biotic stresses (Mouradov and Spangenberg 2014). Terpenoids are also involved in plant-pathogen interactions (Jansen et al. 2011). In grapevine, they may be involved in defense against downy mildew (Algarra Alarcon et al. 2015; Chalal et al. 2015). At T0, discriminant masses were mainly related to
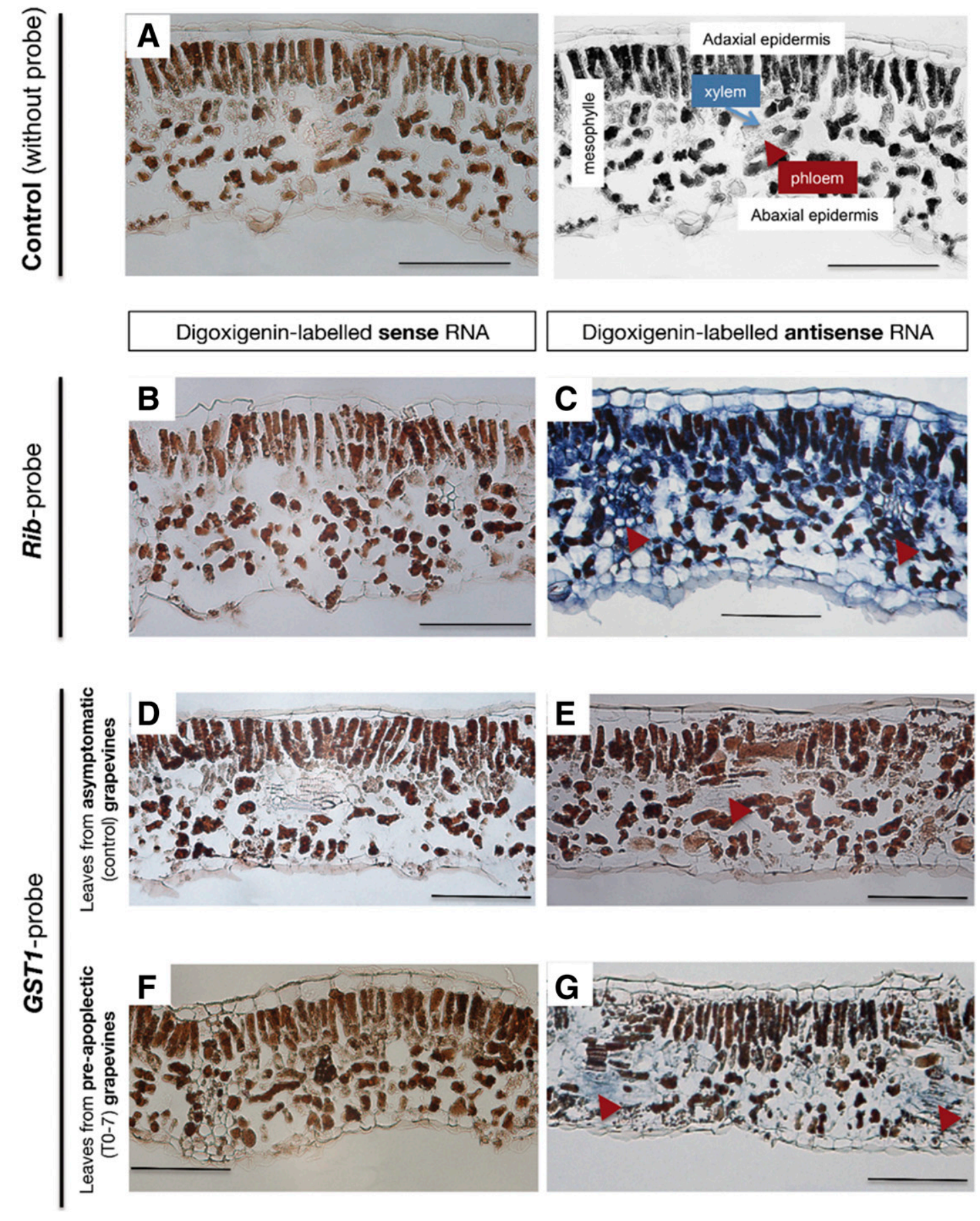

Fig. 7. Visualization of GST1 expression in grapevine leaf tissues. A, A control without probe was made to ensure that colorimetric (blue) signals further observed correspond to a specific detection of the probe. $\mathbf{B}$ to $\mathbf{G}$, The transcripts were localized using a digoxigenin-labeled antisense probe (C, E, G) and the specificity of hybridization was checked with the corresponding sense probe as negative control (B, D, F). VvGST1 localization (E, G) was compared with the $26 \mathrm{~S}$ ribosomic (Rib) RNA probe pattern, which was known to be constitutively expressed in all tissues (C). VvGSTl transcripts were not detected in asymptomatic (control) leaves (E), whereas it was visualized in phloem cells of preapoplectic ones (G, red arrow heads). Bars represent $100 \mu \mathrm{m}$. 
biosynthesis of secondary metabolites, starch and sucrose metabolism, galactose metabolism, phenylpropanoid biosynthesis, and flavonoid biosynthesis metabolisms. The defensive secondary metabolism continues to remain activated, but the terpene one no longer contributed to diseased sample specificity. It seems, therefore, that primary metabolism, mainly related to amino acids, is highly mobilized at T0-20 and T0-7, whereas secondary metabolism is activated at T0. All these results suggest a mobilization of the stress-responsive secondary metabolism and are in accordance with the previously reported activation of the expression of defense genes before apoplexy and their implication during disease progression in leaves (Letousey et al. 2010) and in wood (Magnin-Robert et al. 2014).

As pathogen propagules and byproducts are able to diffuse from the infected woody tissue of the trunk and branches to the crown of the affected vines, some of their metabolites were shown to occur in the xylem sap of naturally infected grapevines
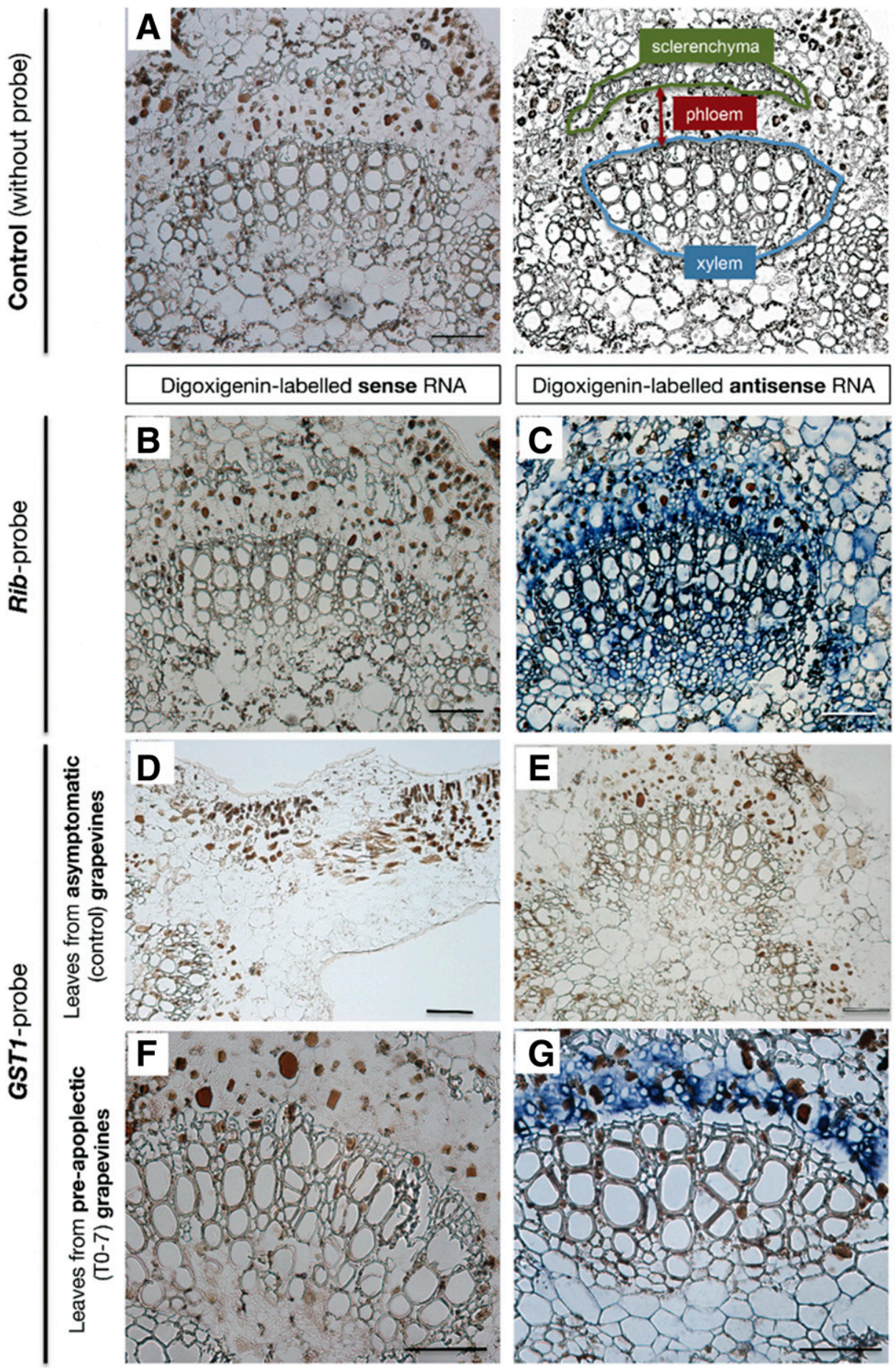

Fig. 8. Visualization of GST1 expression in leaf primary bundles. A, A control without probe was made to be sure that colorimetric (blue) signals further observed correspond to a specific detection of the probe. $\mathbf{B}$ to $\mathbf{G}$, The transcripts were localized using a digoxigenin-labeled antisense probe (C, E and G) and the specificity of hybridization was checked with the corresponding sense probe as negative control (B, D and F). VvGST1 localization (E and G) was compared with the $26 \mathrm{~S}$ ribosomic (Rib) RNA probe pattern, which was known to be constitutively expressed in all tissues (C). VvGSTI transcripts were not detected in primary bundle asymptomatic leaves (E), whereas they appeared to be exclusively localized in phloem cells of preapoplectic ones (G). Bars represent $100 \mu \mathrm{m}$. 
(Bruno et al. 2007). As we are not able to distinguish plant metabolites from microbe metabolites, we cannot exclude the possibility that microbe common metabolites contribute directly to the metabolic signature of the discriminated sample groups. Moreover, esca-associated fungi may also contribute to the evolution of some compounds. For example, previous studies conducted with trees have reported the adaptation capability of fungi to metabolize terpenoids and stilbenes and use them as carbon sources for wood colonization (Diguistini et al. 2011; Hammerbacher et al. 2013). This could also be a hypothesis in esca-affected grapevine, since phytoalexins were not significantly increased in apoplectic leaves as described below.

VK and chemical composition diagrams indicated that sulfurcontaining compounds contributed to the metabolite fingerprint of the sample groups. Sulfur-containing compounds are known to play an important role in the plant response to stress (Capaldi et al. 2015). More specifically, glutathione is probably involved in esca symptom expression (Letousey et al. 2010; Valtaud et al. 2009). In our study, assessment of the evolution of putative glutathione showed a lower accumulation in diseased samples than in control ones, correlated with a higher expression of GSTencoding genes. Valtaud et al. (2009) also reported a lower glutathione content in leaves expressing the chronic form of the disease. However, conversely to what they reported, no mass corresponding to the reduced form of glutathione could be found in our conditions. This might be explained by the difference in the disease expression (apoplectic, chronic). A network analysis allowed the identification of transformations involving glutathione as substrate or its products. As glutathione is known to play a role in the detoxification process, the occurrence of putative glutathione conjugates to toxins previously identified in esca disease was investigated, using their $\mathrm{m} / \mathrm{z}$ values and predicted raw formulas. However, no calculated mass corresponding to putative glutathione-conjugated toxins commonly associated to trunk diseases could be found.

In situ hybridization was performed to localize the GST1 gene. A preferential phloem-specific expression of this gene was observed. In this sense, Fukuda et al. (2004) have reported the activity of a phloem-specific GST in rice leaves. To our knowledge, an immune fluorescein isothiocyanate-labeling targeting two different GST proteins, GSTF and GSTU, has already localized these proteins in veins and particularly in phloem (Valtaud et al. 2009). Moreover, the tau GSTU protein, encoded by the GSTI gene, was mainly found in the plastids (Valtaud et al. 2009), which suggest an important role in detoxification processes to avoid oxidative damage in chloroplasts (Kuźniak and Sklodowska 2001). GST proteins were proposed to detoxify reactive molecules, such as membrane lipid peroxides, by conjugation to glutathione (Marrs 1996) and some GSTs also function as glutathione peroxidases to detoxify reactive oxygen species directly (Bartling et al. 1993). Sieve elements, which have lost most of their organelles, could therefore defend themselves against oxidative stresses by inducing GST activity. Indeed, the expression of GST in the phloem sap of preapoplectic vines could permit the protection of sieve tubes from oxidative damage, especially as this type of stress is frequently described in response to esca (Bertsch et al. 2013). Moreover, GSTs modify toxic compounds into less toxic and more water soluble conjugates (Marrs 1996). Therefore, as discussed by Fukuda et al. (2004) in the case of rice, the presence of GST activity in grapevine phloem sap of preapoplectic individuals suggests the possibility of glutathione conjugation of endogenous compounds in sieve tubes. One of the possible functions of GST in the vascular cells could be in changing hydrophobic compounds into hydrophilic ones conjugated with glutathione, making these modified compounds more mobile in the sieve tubes and more easily distributed along the plant tissues. Otherwise, some plant GSTs showed flavonoid-, auxin-, and cytokinin-binding activities, suggesting that these proteins could act as ligands and support the intracellular transport of flavonoids and plant hormones (Bilang and Sturm 1995; Gonneau et al. 1998; Mueller et al. 2000). In this context, and since phloem represents a way for signaling and defense (van Bel et al. 2013), one could imagine that this contributes toward delivery throughout the plant (since the phloem circulates in an up and down manner) of a long-distance alert message of a physiological disorder. Valtaud et al. (2009) observed an enhanced expression of both GST genes (phi GSTF and tau GSTU1) in plants attacked by esca. The expression of these genes was greatest in leaves that were visually healthy, suggesting that the GSTs are the first enzymes induced in the response to esca.

In this study, we show that transcriptomic and nontargeted metabolomic analyses proved to be very effective in discriminating the profiles of apoplectic and healthy leaves of diseased plants, thus demonstrating the great potential of these tools in understanding the complex GTDs. Among the changes reported, most concern the metabolism of carbohydrates, amino acids, phenylpropanoids, and plant defense responses. Gene expression, such as SucS2, Aux10A5, MYB4A, EpoxHF, and Hahl, could be identified as markers of the preapoplectic event in 'Chardonnay'. Our results support, also, the hypothesis that glutathione could be an early actor during apoplexy appearance, because it is present at a lower level in the apoplectic leaf compared with the control, and that GST1 gene expression is preferentially noted in phloem, suggesting a long-distance transport of glutathione, which reinforces the toxin hypothesis of symptom development. In turn, such markers need to be validated in other cultivars that are more or less susceptible to trunk diseases and, also, in other climatic conditions, since abiotic stresses could affect the plant response.

\section{MATERIALS AND METHODS}

\section{Plant material.}

Experiments were performed on 'Chardonnay' grapevines (Vitis vinifera L.) planted in 1986, grafted on 41B rootstock, and trained according to the Chablis method. The experimental site was located in the Moët and Chandon vineyard in Avize (Champagne region, France). The site is characterized by an average annual temperature of $10.8^{\circ} \mathrm{C}$, reaching an average of $19^{\circ} \mathrm{C}$ during apoplexy expression, and $480.7 \mathrm{~mm}$ of annual precipitation. From 2005 to 2007, two groups of vines were defined in the plot: i) vines that did not show any esca symptoms, visually healthy and considered as unaffected controls, and ii) vines that developed acute chronic or apoplexy symptoms, considered as diseased. In the wood of all plants, the major causal agents of esca disease were previously isolated (Magnin-Robert et al. 2014).

To investigate vines before and during acute esca expression, plants with a high potential to develop the apoplectic form were selected, i.e., plants from the second group that expressed symptoms during the previous summer. Since the first apoplectic form symptoms appear in July in the Champagne region, all measurements and sample harvests were performed twice a week from mid-June to the end of July in both 2008 and 2009. Numerous plants were therefore followed in June but only those expressing apoplexy in July were used for the study. At the appearance of foliar symptoms, a retrospective chronology of the disease could be established, as described by Letousey et al. (2010). The analyzed leaf samples were thus harvested i) 20 days before apoplexy appearance (T0-20) with visually healthy leaves, as controls, ii) 7 days before apoplexy appearance, leaves were either healthy or without symptoms (T0-7vh or T0-7tg), and iii) the day the starting apoplexy symptom expression began (T0), leaves were visually healthy, neither turgorless nor drying. Category T0-7tg leaves were dried at $\mathrm{T} 0$ and were not sampled. In the meantime, leaves 
were collected from asymptomatic (control) plants, i.e., vines from the first group described above.

Harvested leaves were immediately frozen in liquid nitrogen in the vineyard and were then stored at $-80^{\circ} \mathrm{C}$, until extraction. Samples were ground in liquid nitrogen to a fine powder and were prepared differently, according to the analysis performed.

\section{Next Generation sequencing: 454- and Illumina sequencings.}

In this study 454- and Illumina sequencing techniques were combined: i) 454-sequencing was used to constitute a unigene set from the pool of extracted RNA from various conditions and ii) Illumina sequencing was used to create RNA sequence data for each of the treatments; secondly, the Illumina sequences were mapped onto the unigene set previously generated and on genome databases. In the case of 454 sequencing, RNA extraction and cDNA synthesis and size fractionation were realized by Express Genomics Inc. (Frederick, MD, U.S.A.). Total RNA was extracted using the Concert plant RNA reagent (Thermo Fischer Scientific, previously Invitrogen, Carlsbad, CA, U.S.A.), according to the manufacturer's protocol. To generate the cDNA, nucleic molecules were digested with NotI and in vitro RNA transcripts were produced using the SP6 RNA polymerase promoter, according to Express Genomics Inc. adapted protocol. First-strand cDNA was made from these transcripts, using a modified primer adapter that reduces the size of the polyA sequence (to about 20 As). After the second strand synthesized, the double-strand cDNA is blunt-ended and size-fractionated. The 454 library was made by Beckman Coulter Genomics (Danvers, MD, U.S.A.) and was created by ligating the Roche titanium adaptators on the Beckman Coulter SPRI-TE instrument. Sequencing was performed on the Genome Sequencer FLX with GS FLX Titanium series reagents, using 35,000,000 beads. In the case of Illumina sequencing, also realized by Beckman Coulter Genomics, cDNA was generated from polyA ${ }^{+}$-purified total RNA (Illumina mRNA sequencing sample preparation guide; Illumina, Inc., San Diego, CA, U.S.A.), followed by adaptator ligation on the Beckman Coulter SPRI-TE Instrument. Sequencing was performed on the Illumina HiSeq2000 instrument at $1 \times 50$ bp read length, using v3 chemistry.

\section{Read mapping, assembly, and differential-expression analysis.}

The mapping assembly was performed with MIRA version 3 , with the 454 sequences and the consensus sequences compiled with the University of Harvard Vitis vinifera Gene Index tool (from 350,000 available expressed sequence tags). In addition, the Illumina mapping was done with Eland program (CASAVA v1.7.0). For amelioration of transcript annotation, BLASTx on the National Center for Biotechnology Information COG (clusters of orthologous groups) database was used. The mapping of Illumina sequences on the genome was done with the software TopHat. Analyses of mapping were performed with software Cufflinks, Cuffcompare, and Cuffdiff, with reference to the data gtf and fasta form EnsemblePlants. The Cuffdiff analyses were made chronologically and separately between the control and the diseased plant for detecting genes whose expression varies during the follow kinetics. Gene expression was considered as significantly up- or downregulated to the control when changes in relative expression were $>2$ or $<0.5$.

\section{qRT-PCR analysis.}

The analysis was performed on leaves of diseased and control plants collected at timepoints T0-20, T0-7, and T0, as for other analyses. Comparing to the Illumina study, some of the samples used were the same and others were obtained from diseased plants $(n=3)$ cultivated in the same vineyard during the same year. Moreover, leaves were also collected at T0-50 and T0-30, in order to extend the kinetic study. Total RNA was isolated, using a Plant RNA purification reagent (Invitrogen, Cergy Pontoise, France), according to Magnin-Robert et al. (2011). In total $150 \mathrm{ng}$ of total RNA was reverse-transcribed, using Verso cDNA synthesis kit (Thermo Fischer Scientific, Surrey, U.K.) according to the manufacturer's protocol. PCR conditions were those described by Bézier et al. (2002). The expression of 18 genes selected from transcriptomic analysis was tracked by qRT-PCR using the newly designed primers. The data were analyzed using CFX Manager software, and the relative levels of gene expression were determined, following the method of Hellemans et al. (2007), with EF1- $\alpha$ and 60SRP as internal reference genes. Results represent the relative expression of genes in leaves of preapoplectic plants at timepoints T0-50, T0-30, T0-20, T0-7, and T0 versus controls at corresponding timepoints. The analyzed genes were considered significantly up-or downregulated when expression changed was $>2$ or $<0.5$, respectively.

\section{FT-ICR-MS analysis.}

A 15-mg sample of each leaf was suspended in $1 \mathrm{ml}$ of methanol (LC-MS grade) (Fluka Analytical, Sigma-Aldrich, St. Louis) and was sonicated for $30 \mathrm{~min}$. After centrifugation $(25,000 \times g, 10 \mathrm{~min}$, room temperature), the supernatant was collected and diluted in methanol $(1: 50 \mathrm{vol} / \mathrm{vol})$ prior to analysis. Ultrahigh-resolution mass spectra were acquired using an FT-ICR-MS (solariX, Bruker Daltonics GmbH, Bremen, Germany) equipped with a 12 Tesla superconducting magnet (Magnex Scientific Inc., Yarnton, U.K.) and an APOLO II ESI source (Bruker Daltonics $\mathrm{GmbH}$ ) operated in the negative ionization mode. Samples were introduced into the microelectrospray source at a flow rate of $120 \mu \mathrm{h} \mathrm{h}^{-1}$. Spectra were acquired with a time domain of $4 \mathrm{M}$ words over a mass range of $\mathrm{m} / \mathrm{z} 100$ to 1,000 and 300 scans were accumulated per sample. Spectra were externally calibrated on clusters of arginine $(10 \mathrm{mg}$ per liter, in methanol). Further internal calibration was performed for each sample by using a list of ubiquitous fatty acids and recurrent wine compounds, allowing mass accuracies of $0.1 \mathrm{ppm}$ (Gougeon et al. 2009). The $m / z$ peaks with a signal-to-noise ratio (S/N) of $\geq 4$ were exported to peak lists. Exact masses were then subjected to Netcalc algorithm and an in-house software tool to obtain chemical formulas (Tziotis et al. 2011). Elemental formulas attributed to $\mathrm{m} / \mathrm{z}$ values were next represented using two-dimensional VK diagrams, which sort values onto two axes according $\mathrm{H} / \mathrm{C}$ and $\mathrm{O} / \mathrm{C}$ atomic ratios (Tziotis et al. 2011). Their distribution according to their elemental compositions $(\mathrm{CHO}, \mathrm{CHOS}, \mathrm{CHON}$, CHONS, CHOP, CHONP, CHONSP) was represented using histograms. All the processes were done with SIMCA-P+12 (Umetrics, Umea, Sweden). The metabolic pathways associated

Table 2. Primers used to generate template DNA for synthesis of RNA probes

\begin{tabular}{lllc}
\hline Gene & Accession no. & \multicolumn{1}{c}{ Primer sequences } & Product (bp) \\
\hline Glutathione- $S$ transferase $(G S T 1)$ & AY156048 & $5^{\prime}$-TTCGAAACTTTTGGGAAGTTCAAC-3' & 243 \\
26S rRNA & AY847628.1 & $5^{\prime}$-TCAAACAACCGCAATAATATCCCT-3' & $5^{\prime}$-CCGACCTCGATCTTATGAGAAGGG-3' \\
& & $5^{\prime}$-CCAAGTCAGACGAACGATTTGCACG-3' & 150 \\
\hline
\end{tabular}


to selected masses were obtained by KEGG query with Vitis vinifera as the organism, using the web server MassTRIX. Network analysis performed from the complete data set and the sulfur-containing compounds was performed as previously described (Adrian et al. 2017). Nodes represent $\mathrm{m} / \mathrm{z}$, values (metabolite candidates) and edges represent chemical reactions. The statistical occurrence of each reaction (mass differences) was tracked through the network and the frequencies of these reactions associated to each group are indicated in terms of $\mathrm{z}$-scores; $\mathrm{z}$-scores $>1.9$ correspond to $P$ values $<0.05$ and $\mathrm{z}$-scores $>2.6$ correspond to $P$ values $<0.01$.

OPLS-DA was applied to the dataset to discriminate sample groups. The overfitting was checked by the $P$ value calculated with cross-validation analysis of variance. From the classification models, the $m / z$ values with the highest regression coefficient were extrapolated in different lists as representative masses for the different clusters (top $\mathrm{m} / \mathrm{z}$ ). These analyses were performed with SIMCA-P+112 (Umetrics).

\section{In situ hybridization analysis.}

In situ hybridization was carried out on leaf samples collected on control plants and on preapoplectic (turgorless and visually healthy) ones at T0-7. Samples were fixed into a fresh mixture of $4 \%$ paraformaldehyde in potassium phosphate buffer $(10 \mathrm{mM}, \mathrm{pH} 7.4)$ and were then embedded in paraffin (Colas et al. 2010). Wax sections at $10 \mu \mathrm{m}$ thickness were obtained using a microtome and were deposited on silanized slides. Primers were designed for PCR amplification of specific regions of GST1 cDNAs (Table 2) and 26S ribosomic RNA sequence (Rib) was used as control. Sense and antisense probes were labeled according to Colas et al. (2010). To increase signals of the GST probe, the DIG oligonucleotide tailing kit (Roche, Indianapolis, IN, U.S.A.) was used to add poly-Dig UTP tail at the 3' end of the amplified RNA sequence (Garcia et al. 2013). Hybridization was performed as previously described by Colas et al. (2010).

\section{ACKNOWLEDGMENTS}

This work was funded by the interprofessional comity of vine in France (CNIV) and especially supported by those of the Champagne (CIVC) and Burgundy areas (BIVB). The company Moët \& Chandon is thanked for making available the vineyard used as the experimental plot in this study.

\section{LITERATURE CITED}

Adrian, M., Lucio, M., Roullier-Gall, C., Héloir, M. C., Trouvelot, S., Daire, X., Kanawati, B., Lemaître-Guillier, C., Poinssot, B., Gougeon, R., and Schmitt-Kopplin, P. 2017. Metabolic fingerprint of PS3-induced resistance of grapevine leaves against Plasmopara viticola revealed differences with elicitor-triggered defenses. Front. Plant Sci. 8:101

Algarra Alarcon, A., Lazazzara, V., Cappellin, L., Bianchedi, P. L., Schuhmacher, R., Wohlfahrt, G., Pertot, I., Biasioli, F., and Perazzolli, M. 2015. Emission of volatile sesquiterpenes and monoterpenes in grapevine genotypes following Plasmopara viticola inoculation in vitro. J. Mass Spectrom. 50:1013-1022.

Bartling, D., Radzio, R., Steiner, U., and Weiler, E. W. 1993. A glutathione $S$-transferase with glutathione-peroxidase activity from Arabidopsis thaliana: Molecular cloning and functional characterization. Eur. J. Biochem. 216:579-586.

Bertsch, C., Ramirez-Suero, M., Magnin-Robert, M., Larignon, P., Chong, J., AbouMansour, E., Spagnolo, A., Clément, C., and Fontaine, F. 2013. Grapevine trunk diseases: Complex and still poorly understood. Plant Pathol. 62:243-265.

Bézier, A., Lambert, B., and Baillieul, F. 2002. Study of defence-related gene expression in grapevine leaves and berries infected with Botrytis cinerea. Eur. J. Plant Pathol. 108:111-120.

Bilang, J., and Sturm, A. 1995. Cloning and characterization of a glutathione S-transferase that can be photolabeled with 5-azido-indole3-acetic acid. Plant Physiol. 109:253-260.

Bruez, E., Lecomte, P., Grosman, J., Doublet, B., Bertsch, C., Fontaine, F., Ugaglia, A., Teissedre, P. L., Da Costa, J. P., Guerin-Dubrana, L., and Rey, P. 2013. Overview of grapevine trunk diseases in France in the 2000s. Phytopathol. Mediterr. 52:262-275.
Bruno, G., Sparapano, L., and Graniti, A. 2007. Effects of three escaassociated fungi on Vitis vinifera L.: IV. Diffusion through the xylem of metabolites produced by two traceiphilous fungi in the woody tissue of grapevine leads to esca-like symptoms on leaves and berries. Physiol. Mol. Plant Pathol. 71:106-124.

Calzarano, F., Di Marco, S., D’Agostino, V., Schiff, S., and Mugnai, L. 2014. Grapevine leaf stripe disease symptoms (esca complex) are reduced by a nutrients and seaweed mixture. Phytopathol. Mediterr. 53:543-558.

Camps, C., Kappel, C., Lecomte, P., Léon, C., Gomès, E., CoutosThévenot, P., and Delrot, S. 2010. A transcriptomic study of grapevine (Vitis vinifera cv. Cabernet-Sauvignon) interaction with the vascular ascomycete fungus Eutypa lata. J. Exp. Bot. 61:1719-1737.

Capaldi, F. R., Lupino Gratão, P., Reis, A., Lima, L. W., and Azevedo, R. A 2015. Sulfur metabolism and stress defense responses in plants. Trop. Plant Biol. 8:60-73.

Chalal, M., Winkler, J. B., Gourrat, K., Trouvelot, S., Adrian, M., Schnitzler, J. P., Jamois, F., and Daire, X. 2015. Sesquiterpene volatile organic compounds (VOCs) are markers of elicitation by sulfated laminarine in grapevine. Front. Plant Sci. 6:350.

Colas, S., Jacquens, L., Manteau, S., Devy, J., Conéjéro, G., Clément, C., Baillieul, F., Mazerat-Gourbeyre, F., and Monti-Dedieu, L. 2010. Expression analysis in grapevine by in situ hybridization and immunochemistry. Pages 361-374 in: Methodologies and Results in Grapevine Research. S. Delrot H. Medrano, E. Or, L.Bavaresco, S. Grando, eds. Springer Netherlands, Amsterdam.

De la Fuente, M., Fontaine, F., Gramaje, D., Armengol, J., Smart, R., Nagy, Z. A., Borgo, M., Rego, C., and Corio-Costet, M. F. 2016. Grapevine Trunk Diseases, a Review, 1st ed. The International Organization of Vine and Wine Publications, Paris, France.

DiGuistini, S., Wang, Y., Liao, N. Y., Taylor, G., Tanguay, P., Feau, N., Henrissat, B., Chan, S. K., Hesse-Orce, U., Alamouti, S. M., Tsui, C. K., and Docking, R. T., Levasseur, A., Haridas, S., Robertson, G., Birol, I., Holt, R. A., Marra, M. A., Hamelin, R. C., Hirst, M., Jones, S. J., Bohlmann, J., and Breuil, C. 2011.Genome and transcriptome analyses of the mountain pine beetle-fungal symbiont Grosmannia clavigera, a lodgepole pine pathogen. Proc. Natl. Acad. Sci. U.S.A. 108:2504-2509.

Fontaine, F., Pinto, C., Vallet, J., Clément, C., Gomes, A., and Spagnolo, A. 2015. The effects of grapevine trunk diseases (GTDs) on vine physiology. Eur. J. Plant Pathol. 104:707-721.

Fukuda, A., Okada, Y., Suzui, N., Fujiwara, T., Yoneyama, T., and Hayashi, H. 2004. Cloning and characterization of the gene for a phloem-specific glutathione $S$-transferase from rice leaves. Physiol. Plant. 120:595-602.

Garcia, K., Haider, M. Z., Delteil, A., Corratgé-Faillie, C., Conéjero, G., Tatry, M. V., Becquer, A., Amenc, L., Sentenac, H., Plassard, C., and Zimmermann, S. 2013. Promoter-dependent expression of the fungal transporter HcPT1.1 under Pi shortage and its spatial localization in ectomycorrhiza. Fungal Genet. Biol. 58-59:53-61.

Gonneau, M., Mornet, R., and Laloue, M. 1998. A Nicotiana plumbaginifolia protein labeled with an azido cytokinin agonist is a glutathione S-transferase. Physiol. Plant. 103:114-124.

Gougeon, R. D., Lucio, M., Frommberger, M., Peyron, D., Chassagne, D., Alexandre, H., Feuillat, F., Voilley, A., Cayot, P., Gebefügi, I., Hertkorn, N., and Schmitt-Kopplin, P. 2009. The chemodiversity of wines can reveal a metabologeography expression of cooperage oak wood. Proc. Natl. Acad. Sci. U.S.A. 106:9174-9179.

Gruau, C., Trotel-Aziz, P., Villaume, S., Rabenoelina, F., Clément, C., Baillieul, F., and Aziz, A. 2015. Pseudomonas fluorescens PTA-CT2 triggers local and systemic immune response against Botrytis cinerea in grapevine. Mol. Plant-Microbe Interact. 28:1117-1129.

Hammerbacher, A., Schmidt, A., Wadke, N., Wright, L. P., Schneider, B., Bohlmann, J., Brand, W. A., Fenning, T. M., Gershenzon, J., and Paetz, C. 2013. A common fungal associate of the spruce bark beetle metabolizes the stilbene defenses of Norway spruce. Plant Physiol. 162:1324-1336.

Hatmi, S., Trotel-Aziz, P., Villaume, S., Couderchet, M., Clément, C., and Aziz, A. 2013. Osmotic stress-induced polyamine oxidation mediates defence responses and reduces stress-enhanced grapevine susceptibility to Botrytis cinerea. J. Exp. Bot. 65:75-88.

Hellemans, J., Mortier, G., De Paepe, A., Speleman, F., and Vandesompele, J. 2007. qBase relative quantification framework and software for management and automated analysis of real-time PCR data. Genome Biol. 8:R19.

Hofstetter, V., Buyck, B., Croll, D., Viret, O., Couloux, A., and Gindro, K. 2012. What if esca disease of grapevine were not a fungal disease? Fungal Divers. 54:51-67.

Jansen, R. M. C., Wildt, J., Kappers, I. F., Bouwmeester, H. J., Hofstee, J. W., and van Henten, E. J. 2011. Detection of diseased plants by analysis of volatile organic compound emission. Annu. Rev. Phytopathol. 49:157-174. 
Koussa, T., Cherrad, M., Zaoui, D., and Broquedis, M. 1998. Composition et tenru en acides gras des feuilles de Vitis vinifera L. var. Cabernet Sauvignon atteintes d'eutypiose. J. Int. Sci. Vigne Vin 32:11-16.

Kuźniak, E., and Sklodowska, M. 2001. Ascorbate, glutathione and related enzymes in chloroplasts of tomato leaves infected by Botrytis cinerea. Plant Sci. 160:723-731.

Lambert, C., Khiook, I. L., Lucas, S., Télef-Micouleau, N., Mérillon, J. M., and Cluzet, S. 2013. A faster and a stronger defense response: One of the key elements in grapevine explaining its lower level of susceptibility to esca? Phytopathology 103:1028-1034.

Larignon, P., Fulchic, R., Cere, L., and Dubos, B. 2001. Observation on black dead arm in French vineyards. Phytopathol. Mediterr. 40S: 336-342.

Letousey, P., Baillieul, F., Perrot, G., Rabenoelina, C., Boulay, M., VaillantGaveau, N., Clément, C., and Fontaine, F. 2010. Early events prior to visual symptoms in apopletic form of esca disease. Phytopathology 100:424-431.

Lima, M. R. M., Felgueiras, M. L., Graça, G., Rodrigues, J. E. A., Barros, A., Gil, A. M., and Dias, A. C. P. 2010. NMR metabolomics of esca disease-affected Vitis vinifera cv. Alvarinho leaves. J. Exp. Bot. 61: 4033-4042.

Magnin-Robert, M., Letousey, P., Spagnolo, A., Rabenoelina, F., Jacquens, L., Mercier, L., Clément, C., and Fontaine, F. 2011. Leaf strip of esca induces alteration of photosynthesis and defence reactions in presymptomatic leaves. Funct. Plant Biol. 38:856-866.

Magnin-Robert, M., Spagnolo, A., Alayi, T. D., Cilindre, C., Mercier, L., Schaeffer-Reiss, C., Van Dorsselaer, A., Clément, C., and Fontaine, F. 2014. Proteomic insights into changes in wood of Vitis vinifera L. in response to esca proper and apoplexy. Phytopathol. Mediterr. 53: 168-187.

Magnin-Robert, M., Spagnolo, A., Boulanger, A., Joyeux, C., Clément, C., Abou-Mansour, E., and Fontaine, F. 2016. Changes in plant metabolism and accumulation of fungal metabolites in response to esca proper and apoplexy expression in the whole grapevine. Phytopathology 106:541-553.

Marrs, K. A. 1996. The functions and regulation of glutathione Stransferases in plants. Annu. Rev. Plant Physiol. Plant Mol. Biol. 47: 127-158.

Moritz, F., Kaling, M., Schnitzler, J.P., and Schmitt-Kopplin, P. 2017. Characterization of poplar metabotypes via mass difference enrichment analysis. Plant Cell Env. 40:1057-1073.

Mouradov, A., and Spangenberg, G. 2014. Flavonoids: A metabolic network mediating plants adaptation to their real estate. Front. Plant Sci. 5:620.

Mueller, L. A., Goodman, C. D., Silady, R. A., and Walbot, V. 2000. AN9, a petunia glutathione $\mathrm{S}$-transferase required for anthocyanin sequestration, is a flavonoid-binding protein. Plant Physiol. 123:1561-1570.
Mugnai, L., Graniti, A., and Surico, G. 1999. Esca (Black measles) and brown wood-streaking: Two old and elusive diseases of grapevines. Plant Dis. 83:404-418.

Petit, A.-N., Vaillant, N., Boulay, M., Clément, C., and Fontaine, F. 2006. Alteration of photosynthesis in grapevines affected by esca. Phytopathology 96:1060-1066.

Rifai, L. A., Koussa, T., Geny, L., Fassouane, A., Broquedis, M., and Dubos, B. 2005. Evolution des teneurs en polyamines libres et conjuguées dans les feuilles de vigne (Vitis vinifera 'Cabernet Sauvignon') saine et atteinte d'eutypiose. Can. J. Bot. 83:194-201.

Romanazzi, G., Murolo, S., Pizzichini, L., and Nardi, S. 2009. Esca in young and mature vineyards, and molecular diagnosis of the associated fungi. Eur. J. Plant Pathol. 125:277-290.

Romero-Pérez, A. I., Lamuela-Raventós, R. M., Andrés-Lacueva, C., and de La Torre-Boronat, M. C. 2001. Method for the quantitative extraction of resveratrol and piceid isomers in grape berry skins. Effect of powdery mildew on the stilbene content. J. Agric. Food Chem. 49:210-215.

Spagnolo, A., Magnin-Robert, M., Alayi, T. D., Cilindre, C., Mercier, L., Schaeffer-Reiss, C., Van Dorsselaer, A., Clément, C., and Fontaine, F. 2012. Physiological changes in green stems of Vitis vinifera L. cv. Chardonnay in response to esca proper and apoplexy revealed by proteomic and transcriptomic analyses. J. Proteome Res. 11:461-475.

Surico, G., Mugnai, L., and Marchi, G. 2006. Older and more recent observations on esca: A critical overview. Phytopathol. Mediterr. 45:S68-S86.

Tziotis, D., Hertkorn, N., and Schmitt-Kopplin, P. 2011. Kendrick-analogous network visualisation of ion cyclotron resonance Fourier transform mass spectra: improved options for the assignment of elemental compositions and the classification of organic molecular complexity. Eur J Mass Spectrom Chichester 17:415-421.

Valtaud, C., Foyer, C. H., Fleurat-Lessard, P., and Bourbouloux, A. 2009. Systemic effects on leaf glutathione metabolism and defence protein expression caused by esca infection in grapevines. Funct. Plant Biol. 36:260-279.

van Bel, A. J. E., Helariutta, Y., Thompson, G. A., Ton, J., Dinant, S., Ding, B., and Patrick, J. W. 2013. Phloem: The integrative avenue for resource distribution, signaling, and defense. Front. Plant Sci. 4:471.

\section{AUTHOR-RECOMMENDED INTERNET RESOURCES}

Cufflinks, Cuffcompare, and Cuffdiff software: http://cole-trapnell-lab.github.io/cufflinks

MIRA sequence assembly website: http://www.chevreux.org/projects_mira.html

National Center for Biotechnology Information COG database: http://www.ncbi.nlm.nih.gov/COG

TopHat software: https://ccb.jhu.edu/software/tophat/tutorial.shtml

University of Harvard Vitis vinifera Gene Index tool: http://compbio.dfci.harvard.edu/tgi 